\title{
A Review on Recent Advancements in FOREX Currency Prediction
}

\author{
Md. Saiful Islam ${ }^{1}\left(\mathbb{D}\right.$, Emam Hossain $^{2}{ }^{(D}$, Abdur Rahman ${ }^{1}\left(\mathbb{D}\right.$, Mohammad Shahadat Hossain ${ }^{2}(\mathbb{D})$ \\ and Karl Andersson 3,*(i) \\ 1 Department of Computer Science and Engineering, Port City International University, \\ Chittagong 4202, Bangladesh; saifulrahat5@gmail.com (M.S.I.); arrashed@outlook.com (A.R.) \\ 2 Department of Computer Science and Engineering, University of Chittagong, Chittagong 4331, Bangladesh; \\ ehfahad01@gmail.com (E.H.); hossain_ms@cu.ac.bd (M.S.H.) \\ 3 Pervasive and Mobile Computing Laboratory, Luleå University of Technology, S-931 87 Skellefteå, Sweden \\ * Correspondence: karl.andersson@ltu.se; Tel.: +46-910-585364
}

Received: 29 June 2020; Accepted: 28 July 2020; Published: 30 July 2020

check for updates

\begin{abstract}
In recent years, the foreign exchange (FOREX) market has attracted quite a lot of scrutiny from researchers all over the world. Due to its vulnerable characteristics, different types of research have been conducted to accomplish the task of predicting future FOREX currency prices accurately. In this research, we present a comprehensive review of the recent advancements of FOREX currency prediction approaches. Besides, we provide some information about the FOREX market and cryptocurrency market. We wanted to analyze the most recent works in this field and therefore considered only those papers which were published from 2017 to 2019. We used a keyword-based searching technique to filter out popular and relevant research. Moreover, we have applied a selection algorithm to determine which papers to include in this review. Based on our selection criteria, we have reviewed 39 research articles that were published on "Elsevier", "Springer", and "IEEE Xplore" that predicted future FOREX prices within the stipulated time. Our research shows that in recent years, researchers have been interested mostly in neural networks models, pattern-based approaches, and optimization techniques. Our review also shows that many deep learning algorithms, such as gated recurrent unit (GRU) and long short term memory (LSTM), have been fully explored and show huge potential in time series prediction.
\end{abstract}

Keywords: foreign exchange (FOREX) market; time series prediction; currency prediction; machine learning

\section{Introduction}

The foreign exchange market, also known as FOREX, is the world's biggest currency exchange market [1] with over $\$ 5.1$ trillion of volume trade per day [2]. It is considered to be very complex and volatile, and is often compared with the black box because of the unknown nature and high fluctuation in currency rates [3]. In the FOREX market, currency trading occurs $24 \mathrm{~h}$ a day [4] but the trading time is divided into four major time zones [5]. These time zones are the Australian zone, Asian Zone, European Zone, and North American Zone. Each of these zones has its own opening hours and closing hours. Currencies are traded in pairs and most traded currencies are priced against the USD [2]. Based on this trade, the foreign exchange market is divided into three different categories: majors, cross-rates, and exotics [6]. Majors are the most traded currencies which are priced against the USD and occupy the majority of the FOREX market. Cross-rates are the currencies that are priced against a currency other than the USD. The last category, exotics, includes all other currencies that are not major but are priced against the USD. Each currency pair has its own opening price, highest price, 
lowest price, and closing price based on the trading session. For security reasons, it is not possible for one person to directly go to, register with, and buy from the FOREX market. A person needs to use a third party, also known as a broker, for buying currencies from FOREX. Brokers are people or companies that have access to the FOREX market and are able to purchase currencies directly from there. In the FOREX market, a person has only two options available, either buying currencies or selling if they have brought any currencies previously. If the selling rate of the currency is larger than the buying rate, it results in profits for the person. However, like the share market, the FOREX market is also dependent on events that affect the economy of a country. That's why traders need to know the behavior of the market before they do their trading.

Recent years have seen a lot of research interest in the FOREX market currency rate prediction. Many researchers have come up with a lot of unique ideas using machine learning for accomplishing this task. Apart from machine learning, many researchers even used the Internet-of-Things (IoT) for estimating rates of different sectors. Such models were used by Salvatore, Vittorio, and Francesco, who used IoT for estimation of a European barrier price [7] and determining the fluctuation and also for estimating the simultaneous buying and selling prices [8]. From statistical to deep learning, different models along with hybrid models have arisen on this occasion. Not only are these models diverse, but they are also different from each other. It is also quite a tough task to find which model is better among them, but a shortlist can be made by analyzing each of these models.

In this research, we tried to review the machine learning methods that have been applied in the previous three years, from 2017 to 2019, for FOREX currency price forecasting. The remainder of the paper is arranged as follows. Section 2 includes the interests of the study, Section 3 presents the conceptual framework, and Section 4 discusses the data and methodology that we used. Section 5 presents the classification of the paper. Section 6 includes reviews based on different machine learning approaches. Section 7 discusses the research gaps and future directions. Finally, Section 8 concludes the present study.

\section{Interests of the Study}

People are invested more in trading of currencies nowadays. Among these markets, two of the biggest trading platforms are cryptocurrency trading and the FOREX market. Cryptocurrency trading, an emerging and growing platform of currency trading, is sometimes considered as similar to the FOREX but has some key differences. Cryptocurrency trading is a relative newbie in the market, while FOREX is a large-scaled, well-set practice. In each trade, FOREX often involves third-party brokers, whereas cryptocurrency lacks them. Another drawback of cryptocurrency trading is the unavailing of the liquidity. Although cryptocurrency promises higher returns than FOREX, FOREX provides stable, highly secured, and highly regulated trading compared to cryptocurrency trading.

Along with other fields, predicting the FOREX market has been a key target of investigators over the previous couple of decades. There are two alternative ways to forecast the foreign exchange market: fundamental research and technical research. Fundamental research considers many factors, such as the economy and political state of a country, the reputation of a company, all internal and external trading news, etc. Technical research solely predicts the FOREX market based on a company's historical data, in particular, the highest price, lowest price, opening price, and closing price of a currency and the volume traded on a particular day. There's evidence that people use both of those methods to forecast the exchange rates [9]. The fundamental analysis was used by Frank Westerhoff to find out the effectiveness [10]. They studied the emergence of bubbles, excess volatility, and volatility clustering. They found that currency rate control had the tendency to scale back the volatility and deviations. Technical analysis has seen its fair share of use in recent years as well [11-13]. Research proves that foreign exchange market currencies have some similarities and they are clustered with each other [14]. 
The emergence of artificial neural networks played a huge role in foreign exchange rate prediction. Due to its predictive capabilities, many researchers used neural networks for their prediction models [15-19]. In the past, the evolutionary computation method was a popular choice [20-23] along with fuzzy logic [24-27] which is popular still now. With the passage of time, newer approaches have been applied and newer models have arisen. However, along with the past methods, we need to consider the latest methods that have been arisen recently.

The intention of this study is to provide a better understanding of these models that were used in research on the area of FOREX forecasting. This paper analyzes the research methods and articles which are directly related with FOREX prediction that have been published in the previous three years in "Elsevier", "Springer", and "IEEE Xplore". One of the objectives of this paper is to give an intuition into the recent conducted methods and also provide a future direction for researchers.

\section{Conceptual Framework}

The FOREX market is difficult to predict because of the fluctuation of the price movements. Many researchers have applied different methods for successfully predicting the FOREX market. The most popular methods in the late 1990s were statistical, natural language processing, and neural network techniques. In 1990, Ulrich, Michel, Richard, Olivier, Matthias, and Claude applied the statistical method for intraday analysis of the FOREX market [28]. They used 15 years of data for creating and evaluating their model. In 1996, Bin Zhou used statistical analysis for analyzing tick-by-tick data of FOREX [29]. He proposed a model for explaining negative auto-correlation and volatility estimators for high-frequency data rates. Similarly, many other researchers used statistical techniques [30-32] and these methods are still very popular $=$ because of newly invented statistical algorithms. Natural language processing has seen some usage $[33,34]$ for predicting time series as well. Although not many researchers have applied natural language processing, the results that NLP produced was remarkable. The neural network was an evolutionary discovery and played a huge role in predicting time series, especially foreign exchange market. Many different models have arisen on this occasion as well [35-38].

The present study brings all the related thesis works applied to forecast the FOREX currency rates using these three approaches. Figure 1 shows a basic organization of our research. We have done a keyword-based search on "Springer", "Elsevier", and "IEEE Xplore" using the keyword "FOREX prediction." Then we found 131 papers on Elsevier, 15 papers on IEEE Xlorer, and 190 papers on Springer within 2017-2019. Some of these studies directly predicted FOREX prices, while the other works did not predict FOREX prices. Table 1 shows the total papers from each of the journals. From the those papers, papers that are directly related to predicting FOREX prices were placed under the "Predicts FOREX Price" category. Papers that are not directly related with FOREX price (like gold price prediction, stock price prediction, etc.) were placed under the "Doesn't Predict FOREX Price" attribute. From the papers related to FOREX price prediction, we classified them against whether they got any citations or not. Table 2 shows the citation summary of these papers. Out of total 73 papers, 39 papers has got at least one citation within March 2020. 


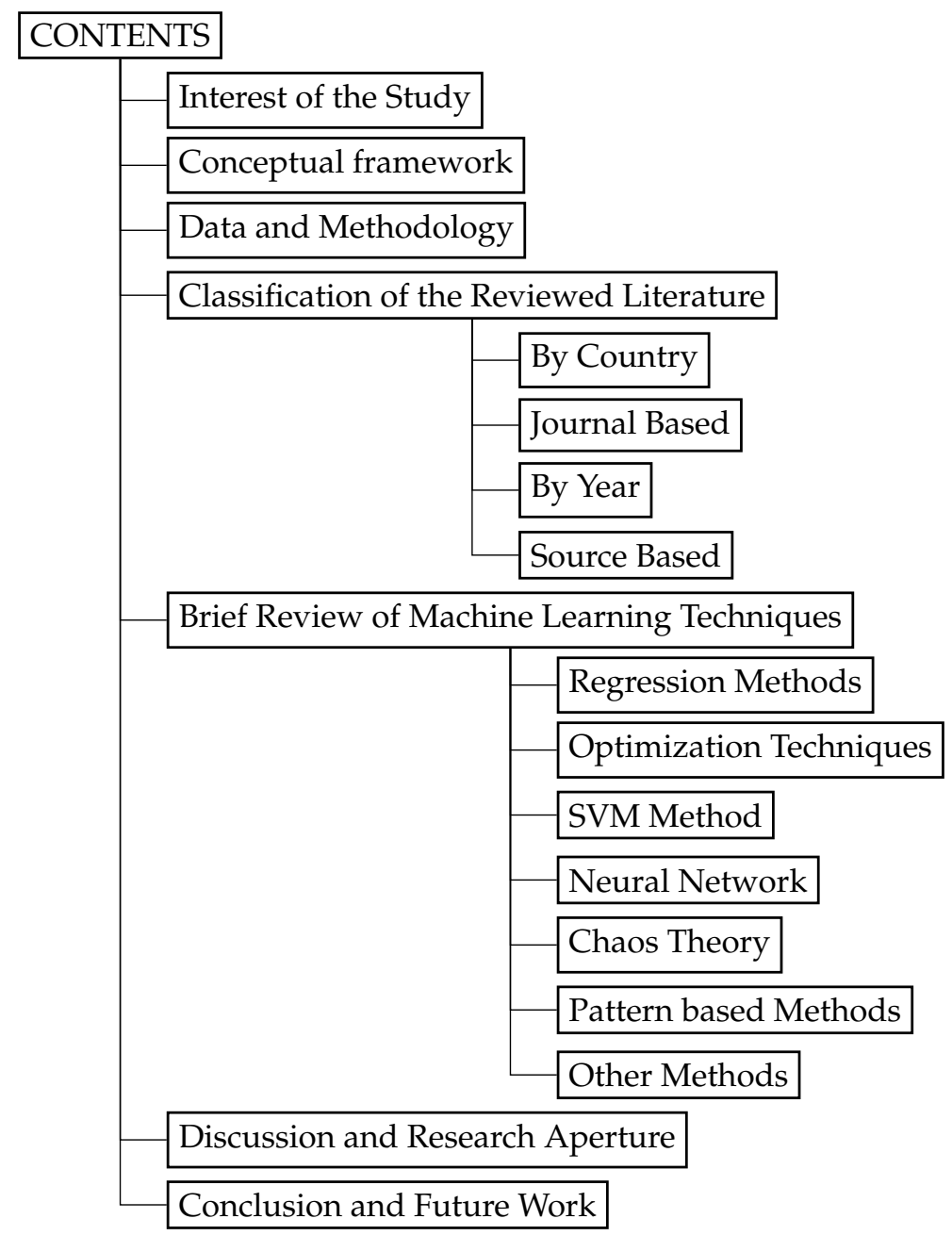

Figure 1. Organization of the research.

Table 1. Summary of the keyword-based search.

\begin{tabular}{cccc}
\hline Journal & Number of Appeared Papers & Predicts FOREX Price & Doesn't Predict FOREX Price \\
\hline Elsevier & 131 & 25 & 106 \\
IEEE Xplore & 15 & 10 & 5 \\
Springer & 190 & 38 & 152 \\
\hline
\end{tabular}

Table 2. Citation received by the papers which predict FOREX prices

\begin{tabular}{cccc}
\hline Journal & Predicts FOREX Price & Citation Count: at Least One & Citation Count: Zero \\
\hline Elsevier & 25 & 19 & 6 \\
IEEE Xplore & 10 & 5 & 5 \\
Springer & 38 & 15 & 23 \\
\hline Total & $\mathbf{7 3}$ & $\mathbf{3 9}$ & $\mathbf{3 4}$ \\
\hline
\end{tabular}

Now we can introduce our algorithm to determine which paper to review in this study: "Select those papers for review which were published on Elsevier, Springer, or IEEE Xplore within the timeframe of 2017-2019, predicted FOREX price, and received at least one citation." Following this algorithm, we found total 39 papers which are reviewed in this research. 


\section{Data and Methodology}

The key objective of this study was to collect, sort out, and gather information on the research on the FOREX forecasting by analyzing the papers. In the past, a lot of researchers used statistical analysis (e.g., Ludmila, Pavel, and Krzysztof [39]; Ardiana, Adhistya, and Silmi [40]; Said, Omar, and Aziz [41]). They used a variety of statistical analysis-based algorithms for forecasting the FOREX currency prices, including regression, decision trees, the trading rule, SVM, and fuzzy systems. Many have used semantic analysis for the same purpose and also got a good result (e.g., Arman, Sayeed, Teh Ying, and David [42]; Saeed and Mehdi [43]). Semantic analysis improves the prediction rate by detecting positive and negative news headlines and predicting accordingly. Most of the other researchers have used the neural network-based models that were capable of predicting time series (e.g., Ranjit, Shruti, Sital, and Subarna [44]; Lina, Yujie, Xiao, Jinquan, Jiguo, and Chengming [45]; Jacek and Piotr [46]). The neural network was an evolutionary discovery since it predicts using hidden neurons and is also capable of adjusting the weighted values which help with providing good accuracy.

This study analyzes the research works published from 2017 to 2019. The research papers issued before this era were not taken into consideration because we wanted to analyze the most recent works and also reflect the researcher's interest in the direction associated to the FOREX. In accordance with the algorithm we have stated in the previous section, this study presents a review of seven papers on neural network methods; four papers each on SVM models, the regression method, and chaos theory; seven papers each on pattern-based methods and optimization methods; and six other papers based on reviews and other not-so-popular techniques that have been published in the given timeframe and were actually relevant to our interests.

\section{Classification of the Reviewed Literature}

Overall, 39 published research articles were scrutinized and categorized by country of origin, year of publication, and source of literature obtained, and this section presents the classification of the scrutinized papers.

\subsection{By Country}

A distribution of the reviewed articles according to the country the research conducted is displayed in Figure 2. Researchers from a large number of countries were interested in this specific field. We considered the research articles from 18 different countries which were published from 2017 to 2019. The highest number of papers (10) were reviewed from India. We reviewed three papers each from Greece, Brazil, and China; and two papers each from Taiwan, Iran, Poland, Portugal, Turkey, and Thailand.

\subsection{Journal Based}

Our reviewed papers were collected from 21 different published journals from Elsevier, Springer, and IEEE. The highest number of papers were reviewed from IEEE conference proceedings (5). The second highest number of papers (4) were collected from Expert Systems with Applications (Elsevier) and Applied Soft Computing (Elsevier) journals. Figure 3 shows the distribution of our reviewed papers according to the journals. 


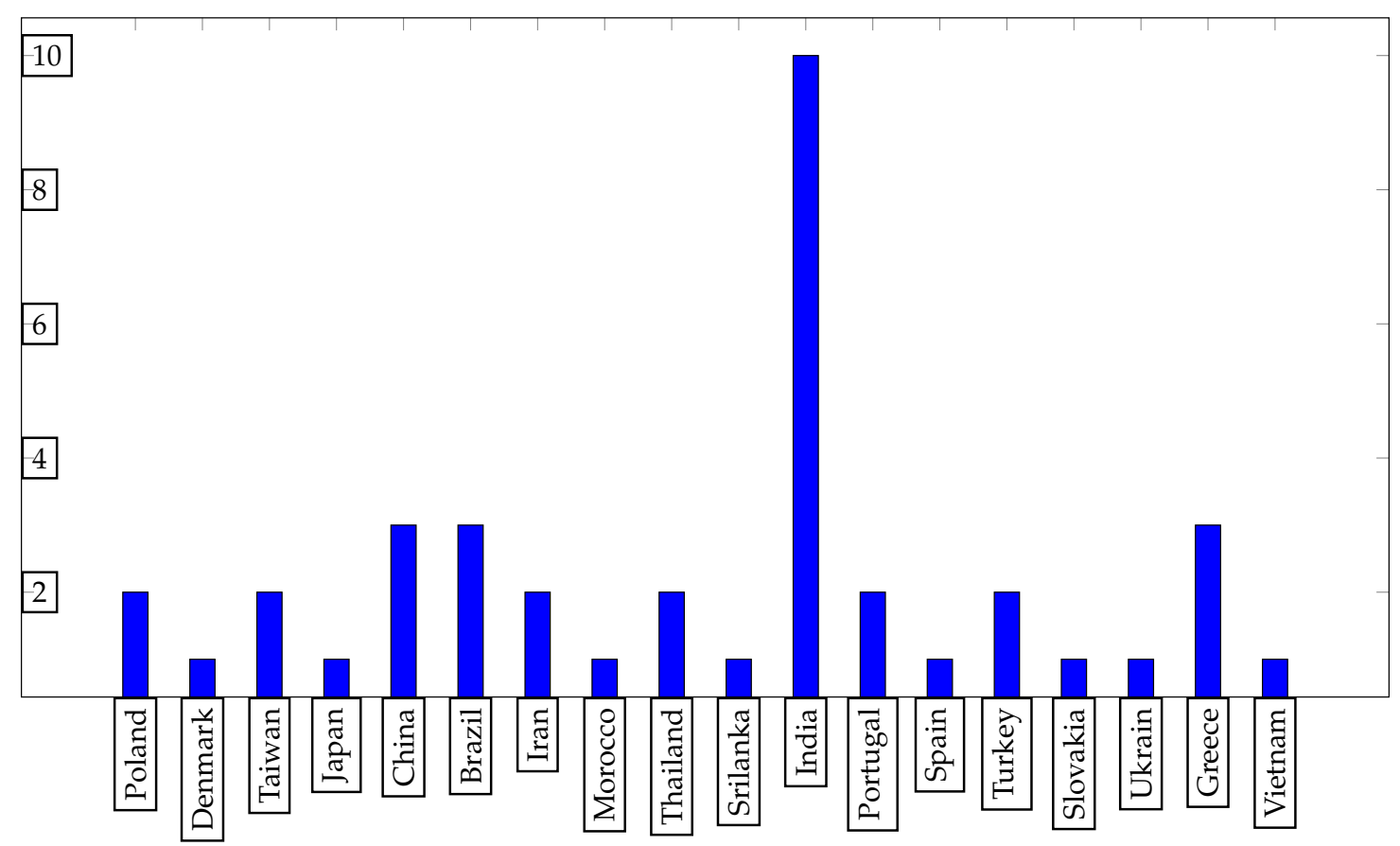

Figure 2. Country-wise classification of studies on foreign exchange market prediction.

\begin{tabular}{ll}
\hline \multicolumn{1}{c}{ Source of Reviewed literature } & Number \\
\hline A. Journals & 4 \\
Expert Systems with Applications & 1 \\
The Journal of Finance and Data Science & 1 \\
Procedia Computer Science & 1 \\
Frontiers of Computer Science & 2 \\
Journal of King Saud University - Computer and Information Sciences & 1 \\
Journal of Intelligent Information Systems & 3 \\
Advances in Intelligent Systems and Computing & 4 \\
Applied Soft Computing & 1 \\
Granular Computing & 2 \\
Neural Computing and Applications & 1 \\
Ubiquitous Communications and Network Computing & 2 \\
Soft Computing & 1 \\
Operations Research Perspectives & 3 \\
Physica A & 1 \\
Vietnam Journal of Computer Science & 1 \\
Swarm and Evolutionary Computation & 1 \\
International Journal of Fuzzy Systems & 1 \\
Computers and Operations Research & 1 \\
Journal of Economic Dynamics \& Control & 2 \\
Knowledge and Information Systems & 5 \\
B. IEEE Conference papers & 39 \\
Total & \\
\hline
\end{tabular}

Figure 3. Sources of reviewed research papers.

\subsection{By Year}

The year-wise assessment of the articles is shown in Figure 4. In accordance with the papers we selected, we reviewed the same number of papers (13 papers) each year. This proves that the research interest in this field has not decreased comparing to the previous years. 


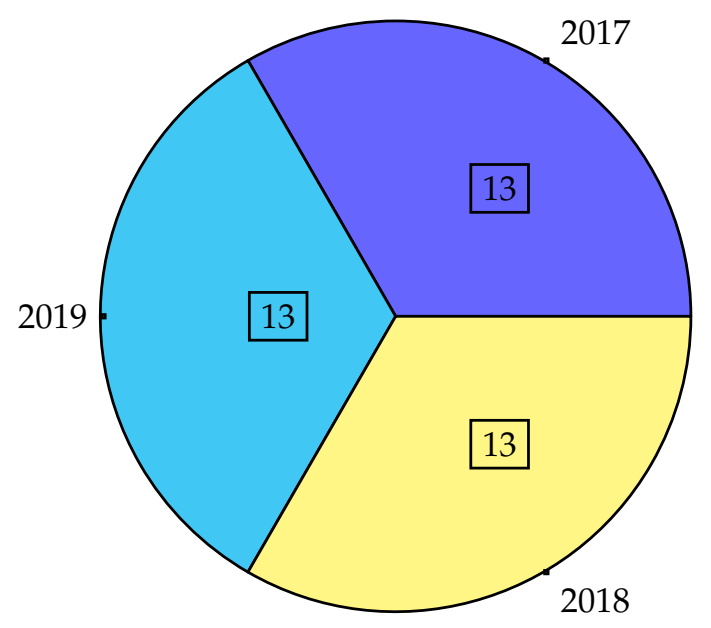

Figure 4. Year-wise categorization of studies on the FOREX market.

\subsection{Source Based}

A lot of papers were published on foreign exchange prediction but we only considered a limited number of them by following a selection algorithm. We only selected FOREX price prediction relevant papers that had at least one citation and was published on Elsevier, Springer, or IEEE Xplore between 2017 and 2019. We can see the distribution of papers according to the journals in Figure 5. As per the collection of the paper from these publishers, almost half of the papers (19) reviewed were from Elsevier. Fifteen papers reviewed were from Springer and five papers reviewed were from IEEE Xplore.

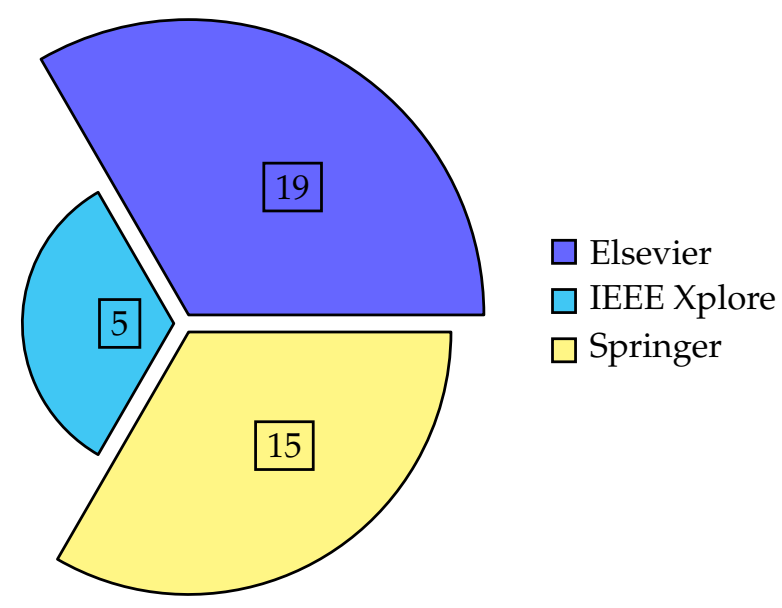

Figure 5. Distribution of papers according to the journals.

\section{Brief Review of FOREX Price Prediction Techniques}

A lot of hybrid methods were tested in previous years. Based on the papers we reviewed, according to the main algorithm the research prioritized, the papers can be split into going along with the following categories: regression methods, SVM, neural networks, optimization techniques, chaos theory, pattern-based methods, and others. These categories were made according to the popularity of the main method of the forecasting system in the past three years. The "Other Methods" category includes algorithms that we did not find in more than one paper among our reviewed articles.

We can get an idea about the past used methods from Bruno, Vinicius, and Herbert's [47] review of machine learning techniques that have been applied to financial market prediction. They reviewed a total of fifty-seven papers related to financial prediction. According to their review, the most referenced 
papers were based on a neural network with 42 references. SVM was placed second with 20 references. Other methods were referenced below 10 times. Figure 6 shows their findings based on references.

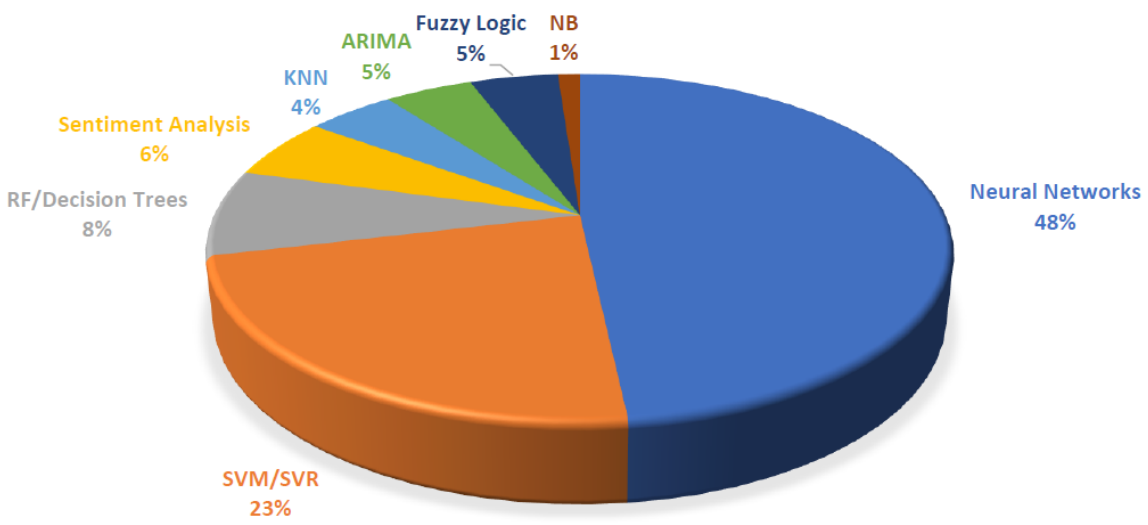

Figure 6. Papers based on references.

The most-cited paper was based on forecasting time series based on SVM with 546 citations and an average of 39 citations per year.

Figure 7 shows the top four methods based on citations according to their papers. The second most-cited paper was based on a neural network with 279 citations and an average of 16.41 citations per year. ARIMA was closely following behind with 278 citations at an average of 23.17 citations per year. Other methods were based on decision tree techniques, sentiment analysis, KNN, ARIMA, and fuzzy logic. Their review concluded that the most used methods for financial prediction were SVM and neural networks.

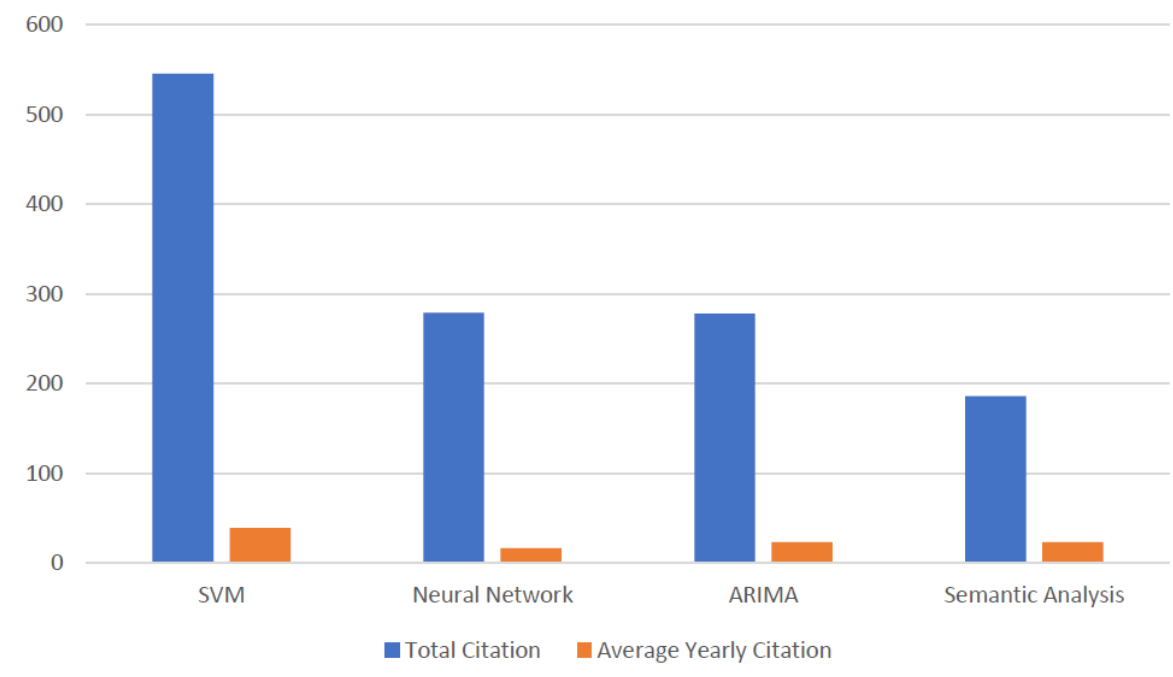

Figure 7. Methods based on citations.

\subsection{Regression Methods}

Milton and Jun [48] proposed a hybrid model for FOREX prediction that uses wavelet models along with support vector regression (SVR). At first, they used a discrete wavelet transform (DWT) method to interpret information from their FOREX dataset. Then the data were used as the input of support vector regression (SVR) for predicting the FOREX prices. They used five currency pairs which include: CHF/JPY, AUD/JPY, GBP/JPY, EUR/JPY, and EUR/CHF. The performances were compared in ME, RMSE, and MAE. They analyzed the performance of their system with ARIMA and ARFIMA 
models. The results showed that their system performs better than ARIMA and ARFIMA models. For AUD-JPY in a 15 min interval, their model obtained $3.1926 \times 10^{-3}$ in ME, $4.6395 \times 10^{-2}$ in RMSE, and $3.4644 \times 10^{-2}$ in MAE.

Paponpat, Kosin, and Nattapol [49] proposed a model for time series inspection and prediction that is based on compressed vector autoregression. At first, they used random compression technique to decrease a large number of FOREX data into a smaller form. After that, they used the Bayesian model averaging (BMA) technique to establish the weight of each random compressed datum to obtain the intersecting parameters. Their model can provide out of sample forecasting till fourteen days prior to the actual time. They used 30 FOREX currency pairs for implementing their system and mean squared error for performance evaluation. Most of the currency pairs had a high mean squared error because the predictors used for forecasting were four lagged dependent variables along with random compression of other FOREX currency pairs. They reached the conclusion that their system was not appropriate to predict all the foreign exchange currencies. Their proposed model proved to have good results for six currency pairs-EUR/TRY, CAD/CHF, EUR/DKK, CAD/JPY, EUR/MXN, and AUD/JPY-and outperformed the existing benchmark of Bayesian autoregression. The CAD/JPY, EUR/DKK, and EUR/TRY currency pairs were best currencies when forecast one day to five days prior; and EUR/CHF, EUR/RUB, and EUR/TRY were the best pairs when forecast eight days to fourteen days ahead of the actual time.

With combining regression techniques along with the cuckoo search algorithm, a hybrid model for speculating USD/EUR variations was developed by the authors of [41]. They proposed a dataset made of JYN, EUR, and BRP variations and USD/EUR currency pair. In order to train their model, they used their dataset with four regression models and also the generated regression weights of these four algorithms as input to the cuckoo search algorithm. Their proposed model presents good results with multiple linear regression.

A huge number of forecasting models were proposed by the authors of the paper [50], by applying linear kernel SVR to historical data for EUROUSD, GBPUSD, and USDJPY currency pairs obtained from high-frequency trading. To forecast the movement of the rates in the next time frame, they used the previous successive timeframes as features. Their datasets contained the finest bid and asking price and the volumes, and in the preprocessed section they removed the volume data along with the asking price. Upon building models, they found a simple rule that provided fine results for systems with single features-that is, return reversal.

There were only four regression techniques that matched our review methodology and have been applied for FOREX prediction in these past three years. After reviewing these four papers, it is clear that support vector regression was the most used algorithm which was included in three papers. Compressed vector autoregression, the CRT regression tree, and partial least squares regression were also used by researchers. However, there are other algorithms such as lasso regression, logistic regression, and multivariate regression which were not explored and can be used for forecasting in later years. The reviewed literature suggests that the system based on a regression model performed better than ARIMA and ARFIMA models [48], and the model performance may increase to a great extent [41] when a regression model is combined with other techniques. However, when working with a large number of currency pairs, it may become difficult with regression techniques, as most of the currency pairs return a higher MSE [49].

\subsection{Optimization Techniques}

Smruti, Debahuti, and Minakhi [51] proposed a forecasting model that combines the Jaya optimization technique with extreme learning machines, which is capable of predicting currency exchange rates. They used two currency pairs, USDEUR and USDINR. They evaluated their proposed model's performance using MAPE, ARV, Theil's U, and MAE. They compared their model's performance with other neural network models. They tested their model against other models based on ELM, NN, and FLANN. They found that regarding ELM, NN, and FLANN, ELM shows the best 
optimization. According to their evaluation data, for MAPE evaluation, ELM DE provides the lowest error. For MAE, ARV, and Theil's U models ELM TLBO, ELM PSO, and ELM Jaya provided the best results respectively.

Spyros and Nikos [52] proposed a technical system for FOREX that was inspired by the Donchian channel method. The main purpose of their strategy was to create profitable portfolios for FOREX trading. They first constructed the modified Renko bars (MRBs) by combining their trading rules. Their modified MRBs proved to be more accurately responsive than the normal candlesticks used in FOREX. They created an optimization stage used by eight currency pairs: NZD/USD, USD/JPY, GBP/USD, EUR/USD, AUD/USD, EUR/JPY, GBP/JPY, and USD/CAD. To achieve their optimization stage, they used three search-derivative-free global optimization techniques. These algorithms were the swarm optimization algorithm, also known as dividing a hyperrectangle (DIRECT), along with multilevel coordinate search (MCS) and pity beetle algorithm (PBA). They tested their optimization strategy and based on the return they constructed two kinds of portfolios: an equally weighted portfolio and a Kelly criterion-based portfolio. They evaluated the performance of their system based on the geometric return, arithmetic mean, and Sharpe ratio. The performance was then matched with the popular Barclay CTA index, S\&P500 benchmarks. They combined the total return of currency pairs on seven years of data from 2010 to 2016; GBP/JPY provided the highest return at $217.59 \%$. EUR/JPY was the second best with $208.74 \%$ total return. The worst was the USD/CAD currency pair with only $29.10 \%$ total return. They found out that the proposed model is not appropriate for three currency pairs: AUD/USD, NZD/USD, and USD/CAD, which provided low returns on their system. Thus, they excluded them and built two additional portfolios: one based on the Kelly criterion and a second equally weighted one, with five currency pairs that had an average return greater than $200 \%$. The result showed that the newly created portfolio based on the Kelly criterion has high stability in its performance and performs better than the old system.

Dadabada and Vadlamani [53] suggested a model for FOREX prediction that was based on a quantile regression neural network and particle swarm optimization. They used PSO to train the QRNN and named the model PSO-QRNN. They used eight currencies: USDINR, EURUSD, GBPUSD and USDJPY, oil price, gold price, NSE India stock index, and standard and poor 500 stock index as datasets for their model. They used seven different algorithms: group method of data handling (GMDH), multilayer perceptron (MLP), random forest (RF), a quantile regression neural network $(\mathrm{QRNN})$, generalized autoregressive conditional heteroskedasticity (GARCH), quantile regression random forest (QRRF), and a general regression neural network (GRNN) for the performance analysis of their model. Their suggested model provided better results than all of the compared models. The results gotten for test data using MSE were as follows: for USD-JPY 0.0000001027, for USD-GBP 0.000000098, for USD-EUR 0.0000000612, for USD-INR 0.000000838, for gold price 0.000002050 , for crude oil 0.00000369 , for S\&P stock 0.000000815, and for NSE Indian stock 0.00000537. When they performed the Diebold-Mariano (DM) evaluation test on all the test results, they found that their proposed PSO-QRNN model performed better than all models on two datasets. For the rest of the datasets, QRRF and QRNN performed better than other models.

Smruti, Kuhoo, Debahuti, and Minakhi [54] proposed a hybrid system that was build using an extreme learning machine's online sequential model and krill herd (KH). The krill herd (KH) was used for optimized feature reduction of the system. They compared their proposed system with a recurrent backpropagation neural network (RBPNN) and extreme learning machine (ELM) algorithm. They considered three factors: (i) without feature reduction (ii) with statistical feature reduction, and (iii) with optimized feature reduction techniques. For optimized feature reduction techniques, they used bacteria foraging optimization (BFO), krill herd, and particle swarm optimization techniques. They used four currency pairs: SGDINR, YENINR, USDEURO, and USDINR for constructing their model. They used 3, 5, 7, 12, and 15 window periods for their system. While evaluating the model's performance, their system showed promising results. For RMSE, MAPE, and Theil'U evaluation, their system performed best with error rates $0.071646,0.10375$, and 0.00014516 respectively. However, 
in MAE and ARV performance evaluations, their proposed model failed to provide the best results. For MAE evaluation, the extreme learning machine's online sequential model, and for ARV evaluation, the krill herd with extreme learning machine (KH-ELM) provided the best results of 0.055458 and 0.00092997 respectively.

For FOREX trading strategy optimization, a genetic algorithm was employed by the authors of the paper [55] to evolve a different set of profitable trading rules inspired from weighted moving average method. They used a time series with 4147 observations within a range of 2001-2015 and they used the close prices of four currency pairs. For generating rules, their genetic algorithm generates returns significantly higher than the exhausted search.

A hybrid machine learning framework was proposed by the authors of the paper [56]. They used a multi-populational Jaya model for predicting the currency exchange rates. They used two FOREX datasets USDEURO and USDINR from the year 2000 to 2016. Their proposed model was matched with the ELM-Jaya and ELM and their model can forecast the currency prices of foreign exchange rates using technical indicators, statistical measures, and combining both of them.

Jui-Sheng Chou et al. [57] represent a sliding window metaheuristic optimization-based prediction system. It is basically a graphically represented interface, created using MATLAB as a standalone application. This system merges metaheuristic intelligence, the novel firefly algorithm, and a least square support vector regressor and was named METAFALSSVR along with a sliding aperture approach. The authors used a benchmark function for verifying optimization effectiveness. For measuring the performance, they studied two cases-daily CANUSD rates and $4 \mathrm{~h}$ EURUSD closing prices, for which they got $0.2532 \%$ and $0.169 \%$ mean absolute percentage errors. The forecast system obtained a rate of accuracy of $89.8 \%$ to $99.7 \%$, which was finer forecasting accuracy than prior models when applied to the CANUSD currency pair comparing to EURUSD exchange price. The error rate of SMOF was $20.8-29.9 \%$, which surpassed the baseline sliding window model.

A total of seven papers were found using our searching methods that are based on optimization techniques. After analyzing these papers, it was found that extreme learning machines (ELMs), the Jaya optimization technique, and the swarm optimization algorithm were equally the most used algorithms. The krill herd $(\mathrm{KH})$ and sliding-window metaheuristic optimization were rarely used. Algorithms such as ant colony, whale optimization, route optimization, gray wolf optimization were not used and can be explored further in the future.

Optimization technique-based systems provide higher ROIs [52] and can be more accurate when combined with powerful techniques - the genetic algorithm, neural networks, regression, etc. [53,55,57]. However, these techniques are not suitable for all currency pairs and may provide better results for only some randomly selected currencies that go well with the models, as we can see in paper [52]. Moreover, some models are dataset-dependent and may perform better only on selected datasets [53].

\subsection{SVM Method}

Bernardo, Rui, and Nuno [58] proposed a model for FOREX market trading that is based on the combination of SVM and the genetic algorithm (GA). SVM classifies FOREX in three different stages while a dynamic genetic algorithm is applied for the trading rule optimization. They trained three variation GA for three different FOREX types using hyper-mutation and adaptability approaches. Then they classified the movements using a rate pattern and optimized the investment strategies using the suitable GA. They used the EUR/USD currency pair as training and evaluation data. They ran the SVM model 50 times; it was trained using 4000 samples. Their model classifies the market into three types: precision, recall, and accuracy. The technical indicator approach showed a higher standard deviation, which may not be reliable for this model. The GA showed a better result compared with other models. They observed that the developed model with leverage performed better than the developed model without leverage. After analyzing their model, they found $83 \%$ return on investment.

Thuy and Vuong [59] proposed a model for FOREX forecasting using SVM. They used the EUR/USD currency pair for their model's implementation. They used the cross validation technique 
for their data and split the results into two categories: positive results and negative results. They used accuracy rate, positive, negative, macro averaging and micro averaging for comparing the performance. Their results show a big difference $(29.5 \%)$ between training sets and testing sets in the Gaussian RBF method, but only a little difference was found in polynomial model. According to the result, the kernel function taken from polynomial theory provides high performance. They compared the normal transaction method with SVM transaction and found that the profit rate was tripled when using an SVM model.

Mustafa, Ismaik, and Onur [60] proposed a model for FOREX prediction called short term movement forecasting in FOREX. Their provided model does not need any human interaction. They found that to locate motif along with learning models such as clustering algorithms and support vector machines to forecast price changes in financial market such as the FOREX market, technical indicator data can be used. The accuracy of the forecasting system was analyzed using different currency pairs. Finally, their result suggests that by using the zig-zag technical indicators to identify motifs which express short-terms trends in FOREX, the result will be higher predicted accuracy along with trade profits.

Mustafaa Onur Ozorhan et al. [61] proposed a model for forecasting the direction and movement of currency rates in the FOREX market. They present a novel approach based on SVM and genetic algorithms for creating a FOREX rate forecasting model. In their system they used different trading ideas and input compositions and obtained different results based on their experiment. Their model provided a variety of accuracies for different pairs. The success of their system highly depends on the selection of learning model, inputs, and decision support mechanisms.

SVM method was used in four papers for predicting the FOREX currencies. The genetic algorithm and SVM hybrid model were the most used techniques. SVMs combined with neural networks have never been explored, so leave a future path open for the researchers. The best feature of the SVM is that it can be used as a classifier [58] and a regressor for forecasting [59,61]. The literature review suggested that when a SVM is incorporated with the genetic algorithm the model can yield a greater ROI [58]. However, sometimes choosing the wrong kernel may provide a huge difference in the result [59]. Furthermore, some systems highly depend on the selection of learning model, inputs, and decision support mechanisms [61].

\subsection{Neural Network}

Rajashree [62] proposed a model that uses a fused recurrent Legendre polynomial neural network and an improved shuffled frog leaping for FOREX prediction. She used a high order neural network with a single layer that is combined with Legendre polynomials along with feedback paths. She applied this model on three different currencies, USDAUD, USDINR, and USDGBP. She used RMSE, MAPE, and MAE for evaluation purposes on her proposed model. She evaluated the RLPNN model with three different expansion orders, 2, 3, and 4. For USDAUD, she observed that expansion order 3 provides a better result. Expansion order 2 provided better results for USDINR and USDGBP. For the RMSE model she used different input sizes and database sizes; she got a good result with aperture size 5 and delay block size 2. For RMSE her proposed model got 0.0268, 0.0258, and 0.0301. For MAPE, 0.6977, 0.4773 , and 0.3331 , and for MAE $0.0303,0.0281$, and 0.0323 for USDAUD, USDINR, and USDGBP respectively. However, due to the expansion order and delay block size, the network size becomes pretty large which is a major drawback of this model.

Lina, Yujie, Xiao, Jinquan, Jiguo, and Chengming [45] proposed a model that predicts the time series of FOREX using the C-RNN method. C-RNN was created using a convolutional neural network and recurrent neural network. They used a data-driven technique to observe the changing characteristics of FOREX. They used the past 10 years' data till 2018 for nine different currency pairs. Their dataset contained 2000 datapoints. They compared the model with an algorithm that was created using a convolutional neural network and long short-term memory. Using RMSE they found that their proposed C-RNN model provides less errors than LSTM and CNN. 
Rajashree [63] proposed a system that is capable of exchange rate prediction using a combination of a computationally efficient functional-link artificial neural network and improved shuffled frog leaping. Improved shuffled frog leaping was used for reducing the error rate of the system. She used three different currencies, USDCAD, USDJPY, and USDCHF for her proposed system. For comparing the systems performance, she used two different algorithms, the particle swarm optimization algorithm and the shuffled frog leaping algorithm. Monthly prediction was done for all three currencies, and RMSE, MAPE, MAE, and MSE were used to analyze the performance. The proposed model, ISFL-CEFLANN, was run 10 times and the performance was recorded for the comparison. Her system provided better results than the compared algorithms. For RMSE the error rate for USD/CAD and USD/JPY currency pairs was between 0.04 and 0.05 and for USD/CHF the range was between 0.03 and 0.04 .

Spyros, Georgios, and Nikos [64] proposed a model called the artificial intelligence risk management system (AIRMS) that is based on machine learning. They developed two risk management systems: One with a neural network (AIRMS-ANN) and another with the decision tree technique (AIRMS-DT). They used five FOREX currencies: EURJPY, GBPUSD, GBPJPY, EURUSD, and USDJPY for implementing those models. They used the technical indicator and past time-series data as the input to their proposed system. They divided the output signal into two categories: profitable and not profitable. When they classified the output signal only as profitable, they got an increase of $50 \%$ profit over the two-category classified model. As evaluation metrics, they used the F1 score for both of their models. Both AIRMS-ANN and AIRMS-DT performed well on average and outperformed each other in some cases. When comparing the Kelly criterion portfolios, the decision tree again beat the neural network in terms of total return.

Rajashree [65] proposed a model that uses a higher order neural network for FOREX prediction. They used a shuffled frog leaping algorithm with the Pi-sigma neural network for predicting dynamic and nonlinear currency exchange rates. Three currencies: USDJPY, USDCAD, and USDCHF were used for implementing their model. The ISFL algorithm was used for estimating the hidden parameters and improving the prediction rate. ISFL algorithm is an improved version of the shuffled frog leaping algorithm (SFLA) wherein convergence the speed of the network is improved along with the predictive ability of the network. They compared the performance of their model with a variety of different models. Their model provided better accuracy along with better statistical performance. For USDJPY, USDCAD, and USDCHF currency pairs the error rates they got using RMSE were 0.0167, 0.0195 , and 0.0210 respectively. Using MAPE the error rates they got were $0.5025,0.2616$, and 0.4476 respectively, and in MAE the error rates were $0.0150,0.0137$, and 0.0171 respectively.

Ehsan, Masoud, Akbar, and Mahdi [66] proposed a new hybrid model for foreign exchange currency price volatility forecasting. They created a new neural network-based system. For building this model, they generated a preset number of imitate data sequences using the GARCH model and other input data variables. They tuned the data sequences and other variables by using particle swarm optimization algorithm to find the best features that provide more accurate prediction of volatility. They used the EUR/USD dataset for the implementation of their model. They found that simulated series created using GARCH model improves the neural network's prediction ability. They used three GARCH models: EGARCH, GJR-GARCH, and GARCH to find the best performing model. While evaluating the 10 day and 15 day prediction data, they found that EGARCH provided the best result in terms of MAPE, MAE, RMSE, and MFE performance evaluation.

Min-Hsuan Fan et al. [67] analyzed the correlation between Taiwan Weighted Stock and Google trend. They used the search volume issued by the Google trend for root testing and finding the connection. Then they analyzed their obtained data and did two experiments, e.g., ML and searching trends. After performing the empirical analysis, the result showed that the support vector machine and decision tree did not perform well as compared with the neural network. Then they compared the $\mathrm{NN}$ with search trends in the second experiment wherein the return value was higher. 
The neural network was quite popular in recent years. A total of seven papers were found on neural networks using our searching method. After analyzing those papers, it was found that different types of neural networks were used by the researchers, such as the recurrent Legendre polynomial neural network and the Pi-Sigma neural network. Many algorithms, such as the modular neural network and deep belief model are yet to be explored. The reviewed literature indicate that neural network-based models can be equipped with different types of approaches, which proves the versatility of these models [62-67]. Moreover, instead of manual audit trails, neural networks have been applied to the financial distress problem, audit fees, internal control risk assessments, etc. [68]. However, in some systems, a large network size may affect the result to a great extent [62].

\subsection{Chaos Theory}

Vadlamani, Dadabada, and Kalyanmoy [69] proposed a three-stage hybrid prediction system that is capable of forecasting the time series successfully. They implemented two different models. First, with chaos, the multilayer perceptron (MLP) and multi-objective particle swarm optimization (MOPSO). Another model was chaos, multilayer perceptron (MLP), and a non-dominated sorting genetic algorithm (NSGA-II). In the first model, they prepared the dataset using minimum lag and minimum embedding dimensions in the first stage. In the second stage, the result produced by stage 1 was fed to a multilayer perceptron (MLP). In stage 3, they tested wheather the chaos was present or not. If chaos was found, the system fed the data to a MOPSO trained auto-regressive model. Otherwise, the polynomial regression was employed in the system. The second model is similar to the first model in the stage 1 and stage 2 but in stage 3 the MOPSO is replaced with NSGA-II. They used JPY/USD, GBP/USD, and EUR/USD currency pairs and gold price (USD) as dataset. They used Thiel's inequality coefficient to find the closeness of the prediction done by their system. Additionally, to figure out whether the predictions were statistically significant or not, they used the Diebold-Mariano (DM) test. According to their result, their proposed second model with chaos, MLP and NSGA-II, outperformed the following hybrids: (i) chaos-MLP-MOPSO, (ii) chaos-GMDH, (iii) chaos-GRNN, (iv) chaos-NSGA-II, (v) chaos-MOPSO, (vi) chaos-PSO, (vii) chaos-MLP-PSO, (viii) chaos-TreeNet, (ix) chaos-MARS, (x) chaos-CART-EB, (xi) chaos-CART, (xii) chaos-RFTE, and (xiii) chaos-LASSO.

Raymond [70] proposed a model for financial prediction based on a chaotic oscillatory multi-agent and neuro-computing system which he named COSMOS. This COSMOS system can be used as a predictor or trading strategy optimization system. The COSMOS forecaster constructed with chaotic FFBP-based supervised learning agents can be used for financial time series prediction, and a COSMOS trader constructed with chaotic RBF-based reinforcement learning agents can be used for trading strategy optimization. He used 2048 trading days consisting of: 84 FOREX currencies, nine cryptocurrencies, 19 commodities, and 17 financial indices' time-series as datasets. After the evaluation of the proposed system, he found that the system provided less than $1 \%$ forecast error for daily prediction of the last 500 days. Additionally, the system provided $8-13 \%$ monthly average returns and outperformed the time-driven strategy and price-driven strategy. He compared the overall performance of the system with the feed-forward back propagation neural network (FFBPN), a SVM, and a deep neural network with principal component analysis (DNN-PCA) for a one-year period. His proposed system outperformed FFBPN, SVM, and DNN-PCA algorithms by $7.49 \%, 3.03 \%$, and $2.67 \%$ respectively.

By using a new hybrid forecasting approach which involves chaos theory (chaos) and multivariate adaptive regression splines (MARS), foreign exchange rates were predicted by the authors of paper [71]. They used chaos theory for constructing state space and MARS for conferring accurate predictions. They tested their system with different types of chaos-based forecasting models for three major FOREX rates: JPY/USD, GBP/USD, EUR/USD, and they got the highest accurate predictions for those FOREX rates with the chaos + MARS approach.

An innovative chaotic interval type-2 fuzzy neuro-oscilattory network (CIT2-FNON) was proposed by the authors of the paper [72], for worldwide financial prediction. The CIT2-FNON 
was constructed by a chaotic discrete-time neural oscillator, namely, the Lee oscillator which serves as transient-fuzzy input neurons of the recurrent networks. The chaotic type- 2 transient fuzzy logic (CT2TFL) was proposed in their model to provide a truly type-2 fuzzy logic system (T2FLS) with a chaotic transient fuzzy property to resolve the complexity problem. In their model CIT2-FNON was integrated with 2048 trading day time series financial data and top-10 major financial signals for the real-time prediction of 129 worldwide financial products.

Four papers were based on chaos theory. By analyzing those papers, it was found that many two and three-stage hybrids based on the chaos theory were implemented in recent years. Among the applied methods, three-stage hybrid chaos + multilayer perceptron (MLP) + non-dominated sorting genetic algorithm (NSGA-II) outperformed all other three-stage hybrids. Research proves chaos's wide applicability as it can be used in a broader way. Reference [70] shows that chaos theory can successfully be used as both a financial time series predictor and as a trading strategy optimizer. One of the most important concerns is choosing the input parameters. The methods are chosen based on the dynamics underlying the chosen data and what kind of analysis is intended for the system. That makes the system highly complex and not always accurate.

\subsection{Pattern Based Methods}

Antonio V., Antonio, Alberto, Sergio, Horacio, Jose J., and Jose M. [73] proposed a new model for FOREX prediction called the elastic network model for FOREX market (ENMX) algorithm that is based on the behavior of macromolecules in dissolution. This model skips local minima and continues to produce unstable values like FOREX and also keeps its distance from an equilibrium. Their model can simulate 21 connected currency pairs and also represent the interactions between a user and system using random movements. They used root mean square error (RMSE) for the performance evaluation of their proposed model. They found that the pseudo-Voigt model is better suited for historical data; hence, they proposed pseudo-Voigt as an alternative distribution for their ENMX model. They compared their model with vector autoregression (VAR) and a driftless random walk model (RW); they found that ENMX performed better than those two compared models. The average RMSE for ENMX was reduced by almost 15\% compared to both VAR and RW models. For average profit, it returned 15\% more profit than VAR; for RW that number was 70\%.

Joao, Rui, and Nuno [74] proposed a system for short term prediction using recurrent reinforcement learning (RL). They used three hidden rectified linear unit neural network layers for their model and trained the model using Q-learning algorithm. They tried something different by using historical tick data to get new state and reward signals. They used only one currency pair EUR/USD as their dataset for training and evaluating their model. They ran their system over 10 times while applying different initial conditions. They found the system's profit results are dataset dependent. They also found that using the validation process, stable candidates can be selected. For normal testing they got an average profit of $78.5+/-9.5$ and for yearly average $11.2+/-1.4$. For the yearly testing result, they got an average total profit of $109.1+/-17.8$ and for a yearly average they got $15.6+/-2.5$. From the triannual testing result, they got an average total profit of $114.0+/-19.6 \%$, and for a yearly average their result was $16.3+/-2.8 \%$.

Erik and Richard [75] proposed multidimensional string models which can be used for time series forecasting. They enhanced the 1-endpoint open string model with a 2-endpoint open string model combined with D2-brane. They showed how the statistics of the predictors can be changed by the new object properties and used them to model a variety of time series systems. They used four different currency pairs in real demo simulations for evaluating their system. After analyzing the result, they found improved net asset value for their proposed model. They also found that higher efficiency for the string models can be achieved by using longer string lengths. As a complementary tool, they also proposed angular momentum to analyze the stability of currency rates.

Paponpat and Nattapol [76] proposed a model to predict the FOREX exchange rate using dynamic model averaging (DMA) and transformed models of general DMA. They applied this model on three 
currency pairs, JPY-USD, EUR-USD, and GBP-USD. Seventy percent of data was used for training the model and the remaining 30 percent was used for the evaluation. They evaluated their proposed model using mean square error (MSE) and mean absolute deviation (MAD). According to the results of their proposed model, they found that autoregression with four lags, also known as AR(4), and time-varying autoregression with four lags, known as the TVP-AR(4) structural break model, provides a better result in USD-JYP prediction. For the EUR-USD dataset the parsimonious model provided good results. They assumed that models that use random walkm which evolves coefficients, are best to use for this prediction. They found reliable consistency for DMA and DMS.

Antoni [77] proposed a system that is based on the Markov chain model and is capable of predicting time series successfully. They used first-order Markov chains and a Markov model of the second-order to find out the best window length, the actual number of windows, and the total number of intervals to increase the predictive efficiency of the system. They considered two types of time series data: EUR/USD one-hour currency pair and WIG20 one-day data, which is a Polish stock exchange index. The performance was measured using the Calmar ratio which shows excellent performance from the system. They used EUR/USD with a candle of one hour, for 50,000 candles. The found a final profit of 7079 pips for 50,000 candles, where pips refer to the price change in the fourth decimal place. They also reached the decision that it is not a guaranteed system that can bring future success, and investing for longer periods is often profitable for stock exchange.

Piotr and Marcin [78] proposed a system wherein they introduced a new approach that was based on the dynamic conditional correlation (DCC). They estimated the parameters by using closing, lowest, and highest rates. They applied the range-based estimator of variance (RBE-V) of returns and the range-based estimator of covariance (RBE-CV) of returns into their DCC model. A range of portfolios of assets were provided by them for the model as it was required by the REB-CV and the suggested model. They compared their system with three different type of existing dynamic conditional correlation models. For GBPUSD, EURUSD, and JPYUSD, the variances gotten were 0.429, 0.238 , and 0.192 and the covariances they got were $0.247,0.422$, and 0.145 respectively.

An algorithmic framework was developed by the authors of paper [79] which is capable of finding, examining, and utilizing new intra-day rate movements in FOREX. They presented a new method for matching patterns and they created more than 16,000 intra-day rate patterns. They used eight different currencies for their experiment. Their predictive accuracy for finding patterns varied between $60 \%$ and $85 \%$.

Pattern-based methods have seen quite a popularity surge in these recent three years and seven papers were found based on this method. After reviewing these papers, it was found that all the algorithms used in these papers were different from each other. Recurrent reinforcement learning (RL), dynamic model averaging (DMA), dynamic conditional correlation (DCC) algorithm, etc., were explored by the researchers. Pattern-based approaches also proved their versatile adapt- ability through their uses in different models [73-79]. Some systems based on patterns can change the statistics of the predictors by using the object properties [75] that can be applied to design a variety of time series systems. However, some pattern-based systems are contradictory to each other, as some models perform well and provide good results with a specific algorithm [76], while other systems perform just the opposite [73]. Additionally, some pattern-based models are only capable of predicting the price over a short-time period and do not guarantee future success for longer period of prediction time [77].

\subsection{Rest of the Methods}

Anastasios, Sotirios, Vasilis, and Nikos [80] proposed a model for FOREX time series prediction by combining a variety of machine learning models predicting results. They used support vector machine (SVM), Bayesian autoregressive trees (BART), random forest (RF), naive Bayes (NB), and dense layer neural network $(\mathrm{NN})$ classifiers for modeling the major currencies' dependency patterns. Depending on the outputs of these algorithms and the information obtained from correlations of their dataset, they created implied signals for exchange price fluctuation. As the last step they turned those 
signals into predictive waveforms using genetic algorithm optimization, leveraging majority voting, and regression weighting techniques. They used the moving-window training approach wherein they tried to imitate the FOREX market's dynamic changes. They took all the major currency pairs for training and testing their system. They used MAE, MAPE, and RMSE for evaluating the performance of their model. For SVM, RF, BART, NN, and AR, using RMSE they got $0.74 \%, 0.73 \%, 0.75 \%, 0.81 \%$, and $0.84 \%$ respectively. Similarly, for MAE, AR performed better than other algorithms. For generating a predictive waveform, the genetic algorithm performs better than other two algorithms. From the results they found that for the USD/CAD currency pair their proposed model performed best and for USD/NZD their system performed the worst.

To select a good set of FOREX experts, an improved expert selection model was proposed by the authors of the paper [81], by learning previously observed sequences. In their model they considered the average mistakes made and average earned by experts, to achieve better performance. They used two real major currency-pair datasets with a period of 29-Apr-2013 to 25-Apr-2014 which consisted of 260 predictions. They proposed their model with good prediction accuracy by RWMAprofit and for profit, the RWMAdual method was efficient.

The context information-based FOREX service model was developed by the authors of the paper [82]. They proposed a C-IOB (context-information, observation, belief) graphical structure model which shows a causal relationship between low-level and high-level context. Their proposed model provides accurate context-aware services and it has the capability to adapt to real-time situations of business needs by deducing belief based on observations. Their simulation results show that the time to execute FOREX transactions is less with context-based beliefs.

Weerathunga and Silva [83] proposed a new hybrid model that combines DRNN and ARIMA models for FOREX prediction. They extracted the input features and used them as the input of DRNN. DRNN predicts the next price value and also feeds the filtered version of data to ARIMA. ARIMA generates the forecast deviation and after combining the results from both DRNN and ARIMA the closing price is obtained. Their model contained three hidden layers, each with 10 nodes, and it was run for 2000 epochs. They used the EUR/USD currency pair data for their proposed model. Their result showed that when the number of epochs or the number of input features increased, the model overfitted and resulted in poor accuracy for the test examples. They concluded that the best result was obtained when they used the current closing price along with the RSI indicator value and EMA indicator values as input features.

A NLP-based model was proposed by the authors [43] using news headlines. They predicted the upward and downward trend of a FOREX currency pair. They focused on determining two things: the proper sentiment of news headlines and proper sense of textual contents to improve the sentiment analysis. They introduced a different novel approach called relevant gloss retrieval, threshold-verb normalization for properly distinguishing the sentiments. They used a sample dataset which contains 15 news headlines with 71 different word pairs and got an accuracy of $83.33 \%$. After increasing the number of news-headlines in their dataset, their new system accuracy was improved to $91.67 \%$.

The rest of the methods contain different types of methods that have been applied for the forecasting of the FOREX market. After analyzing these papers, it was found that Bayesian autoregressive trees (BART), random forest (RF), naive Bayes (NB), ARIMA, etc., were applied and explored by the researchers. Some of these algorithms were applied individually, whereas some were applied in a hybrid model. Natural language processing was rarely explored. Fuzzy logic, the weighted majority algorithm, the weighted moving average algorithm, the generative adversarial network, etc., were not explored by any researchers in this stipulated time. This creates an opportunity for researchers in this field. Some of those techniques achieved better accuracy; however, it would be naive to judge their effectiveness based on one or two works only. Moreover, methods such as NLP based on sentiment analysis that depends on news headlines [43] can easily be misguided using wrong news. Thus, proper security measures need to be applied on these methods. 


\section{Discussion and Research Aperture}

After reviewing 39 research papers, we saw that popular FOREX market predictive approaches in recent years included neural networks, different optimization techniques, and pattern-based approaches, whereas regression methods, SVM models, and chaos theory were the least popular. There are also some papers that did not follow any aforementioned approaches. Figure 8 shows the popularity of the algorithms according to our reviewed papers.

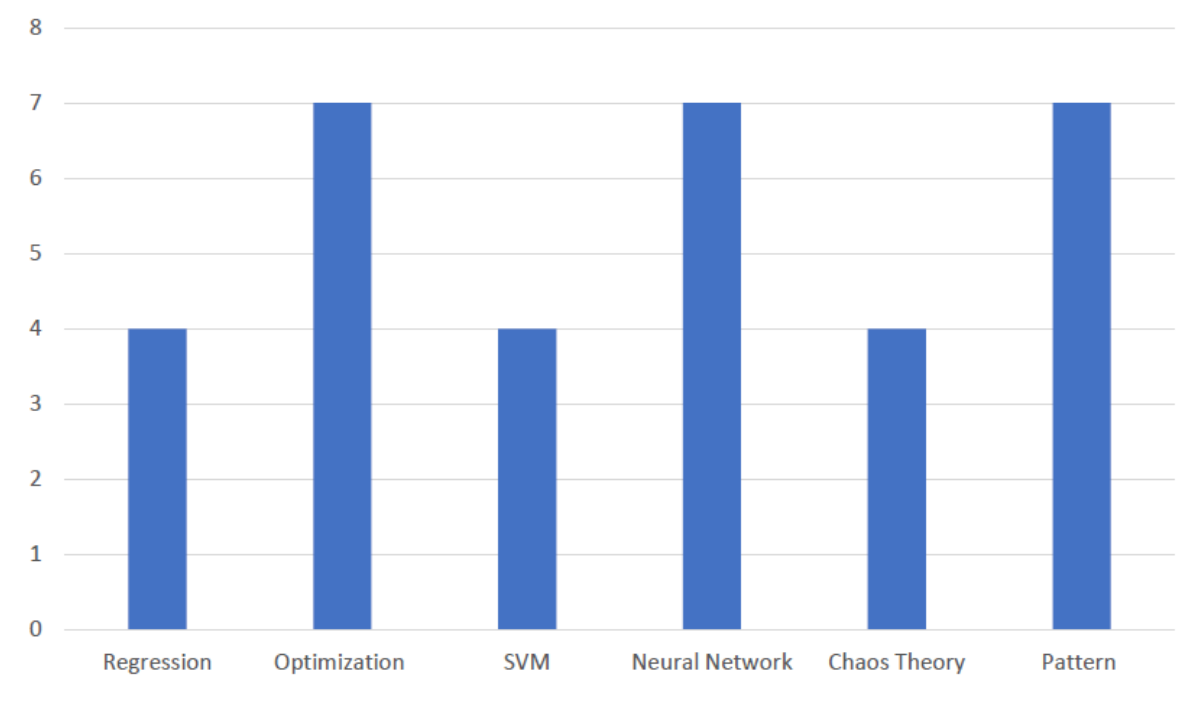

Figure 8. Popularity of the algorithms.

As the unsupervised methods were out of the scope of this research, we cannot compare supervised methods with unsupervised methods. Additionally, many algorithms, such as shrinkage, subset selection, re-sampling methods, and discriminant analysis have not been explored by any of the researchers in recent times. Only a few researchers have shown interest in working with methods such as regression, chaos theory, and SVM-based approaches. Most of the regression methods use a support vector regressor (SVR) for prediction purposes. Some of the researchers used a SVM with another genetic algorithms. Some of them used chaos theory coupled with spline regression. The Markov model, the dynamic conditional co-relation, dynamic model averaging, recurrent reinforcement learning, etc., were chosen by many of the researchers. Some of them used a combination of some predictive statistical approaches for a better performance.

Natural language processing (NLP) provided quite good results with models that used semantic analysis. However, most of the researchers have not shown too much interest in NLP. Recent years have seen only minimal implementation of NLP in the FOREX prediction field. Most of the model was based on news headline recognition using semantic analysis. However, there is a great chance of prediction disasters of these models if false news spreads. That is because these models will analyze and predict according to the given news. However, if the time series of the actual currency rates are applied with semantic analysis, the models will be more secure and will not be affected by false news. NLP has a huge potential to even compete with neural networks. Thus, in future the combination of NLP and neural network can be applied for FOREX rate prediction.

The neural networks with different optimization techniques have seen a huge rise in research interest due to their way of working with greater prediction accuracy. Even though the NN has seen a rise in interest, many neural network algorithms, such as the modular neural network, sequence-to-sequence model, deep belief network, deep feed forward network, deconvolutional network, single multiplicative neural network, and spiking neural network were not even tested in FOREX price prediction within the stipulated time. The group method of data handling (GMDH), the world's first deep learning architecture, was not used by any researchers and the general regression neural network (GRNN) was rarely exploited in this time. The neural network has proved its worth 
for many years now. Most of the high accuracy in predicting time series has been achieved by using neural networks. Each neural network is effective for a different kind of situation. Some perform better in classification; some in normalization. Some work great for predicting time series, whereas some perform average in any kind of situation. The challenge is to use these algorithms efficiently depending on the situation and problem type. Thus, several algorithms can be used in a single model to have a supportive algorithm for different situations. But many algorithms are yet to be explored and many hybrid models are yet to be built.

\section{Conclusions and Future Work}

To analyze the appetite and interests of the researchers in the foreign exchange market, we have reviewed different methods that have been applied for FOREX currency prediction in the last three years (2017-2019). We used a keyword-based search on renowned journal publishers such as Elsevier, Springer, and IEEE Xplore, and developed a selection algorithm to determine which research papers to include in our review. We found 39 papers that satisfy our selection criteria and presented a comprehensive review of these papers. We have discussed the differences between the FOREX market and the cryptocurrency market. From the reviewed literature, it is clear that neural network-based approaches, optimization techniques, and pattern-based approaches were the most liked techniques among the researchers at the aforementioned time. However, it was surprising that natural language processing was not applied vastly compared to other approaches. Multiple linear regression with cuckoo search, SVM with genetic algorithm, CRNN, PSNN with ISFL, and many other methods have been applied in FOREX prediction and have performed quite well. However, many consistent and reliable methods, such as GRU, the Kohonen self-organizing neural network, the modular neural network, and many other algorithms were not fully explored. Many powerful time-series analyzers such as LSTM and GRU have not been fully utilized in this domain. These models can use memory with hidden neurons, which makes them very efficient in time series prediction. This provides a huge scope for research in the near future.

Author Contributions: Conceptualization, M.S.I., E.H., M.S.H., and K.A.; methodology, M.S.I., E.H., M.S.H., and K.A.; software, E.H.; validation, M.S.I.; formal analysis, E.H; investigation, E.H., M.S.H., and K.A.; resources, E.H. and M.S.I.; data curation, E.H. and M.S.I; writing-original draft preparation, M.S.I., A.R., and E.H.; writing-review and editing, E.H., M.S.H., and K.A.; visualization, M.S.I. and A.R.; supervision, E.H., M.S.H., and K.A.; project administration, E.H. All authors have read and agreed to the published version of the manuscript.

Funding: This research received no external funding.

Conflicts of Interest: The authors declare no conflict of interest.

\section{References}

1. Levinson, M. The Economist Guide to Financial Markets: Why They Exist and How They Work; The Economist: London, UK, 2014.

2. Ozturk, M.; Toroslu, I.H.; Fidan, G. Heuristic based trading system on Forex data using technical indicator rules. Appl. Soft Comput. 2016, 43, 170-186. [CrossRef]

3. Anastasakis, L.; Mort, N. Exchange rate forecasting using a combined parametric and nonparametric self-organising modelling approach. Expert Syst. Appl. 2009, 36, 12001-12011. [CrossRef]

4. Huang, R.D.; Masulis, R.W. FX spreads and dealer competition across the 24-h trading day. Rev. Financ. Stud. 1999, 12, 61-93. [CrossRef]

5. Masry, S.; Dupuis, A.; Olsen, R.; Tsang, E. Time zone normalization of FX seasonality. Quant. Financ. 2013, 13, 1115-1123. [CrossRef]

6. Adariani, M.R. Evaluation of the profitability of technical analysis for Asian currencies in the forex spot market for short-term trading. AU-GSB e-JOURNAL 2012, 5, 4-18.

7. Cuomo, S.; Di Somma, V.; Piccialli, F. Pricing estimation of a barrier option in an IoT scenario. Future Gener. Comput. Syst. 2018, 110, 407-412. [CrossRef] 
8. Cuomo, S.; Di Somma, V.; Piccialli, F. A computational method for the European option price in an Internet of Things framework. Future Gener. Comput. Syst. 2017, 107, 730-735. [CrossRef]

9. Lui, Y.H.; Mole, D. The use of fundamental and technical analyses by foreign exchange dealers: Hong Kong evidence. J. Int. Money Financ. 1998, 17, 535-545. [CrossRef]

10. Westerhoff, F. Speculative markets and the effectiveness of price limits. J. Econ. Dyn. Control. 2003, 28, 493-508. [CrossRef]

11. Yen, S.M.F.; Hsu, Y.L. Profitability of technical analysis in financial and commodity futures markets-A reality check. Decis. Support Syst. 2010, 50, 128-139. [CrossRef]

12. Li, J.; Tsang, E.P. Improving Technical Analysis Predictions: An Application of Genetic Programming. In Proceedings of the Twelfth International FLAIRS Conference, Orlando, FL, USA, 1-5 May 1999; pp. 108-112.

13. Sullivan, R.; Timmermann, A.; White, H. Data-snooping, technical trading rule performance, and the bootstrap. J. Financ. 1999, 54, 1647-1691. [CrossRef]

14. Sopranzetti, B.J.; Datar, V. Price clustering in foreign exchange spot markets. J. Financ. Mark. 2002, 5, $411-417$. [CrossRef]

15. Zhang, G.P. Time series forecasting using a hybrid ARIMA and neural network model. Neurocomputing 2003, 50, 159-175. [CrossRef]

16. Chen, A.S.; Leung, M.T. Regression neural network for error correction in foreign exchange forecasting and trading. Comput. Oper. Res. 2004, 31, 1049-1068. [CrossRef]

17. Yu, L.; Wang, S.; Lai, K.K. A novel nonlinear ensemble forecasting model incorporating GLAR and ANN for foreign exchange rates. Comput. Oper. Res. 2005, 32, 2523-2541. [CrossRef]

18. Yu, L.; Wang, S.; Lai, K.K. Adaptive smoothing neural networks in foreign exchange rate forecasting. In Proceedings of the International Conference on Computational Science, Atlanta, GA, USA, 22-25 May 2005; pp. 523-530.

19. Ince, H.; Trafalis, T.B. A hybrid model for exchange rate prediction. Decis. Support Syst. 2006, 42, 1054-1062. [CrossRef]

20. Adamopoulos, A.; Andreou, A.; Georgopoulos, E.; Ioannou, N.; Likothanassis, S. Currency forecasting using recurrent RBF networks optimized by genetic algorithms. In Decision Technologies for Computational Finance; Springer: Berlin/Heidelberg, Germany, 1998; pp. 461-470.

21. El Shazly, M.R.; El Shazly, H.E. Forecasting currency prices using a genetically evolved neural network architecture. Int. Rev. Financ. Anal. 1999, 8, 67-82. [CrossRef]

22. Andreou, A.S.; Georgopoulos, E.F.; Likothanassis, S.D. Exchange-rates forecasting: A hybrid algorithm based on genetically optimized adaptive neural networks. Comput. Econ. 2002, 20, 191-210. [CrossRef]

23. Nag, A.K.; Mitra, A. Forecasting daily foreign exchange rates using genetically optimized neural networks. J. Forecast. 2002, 21, 501-511. [CrossRef]

24. Abraham, A.; Chowdhury, M.U. An intelligent forex monitoring system. In Proceedings of the 2001 International Conferences on Info-Tech and Info-Net, Proceedings (Cat. No. 01EX479), Beijing, China, 29 October-1 November 2001; Volume 3, pp. 523-528.

25. Tseng, F.M.; Tzeng, G.H.; Yu, H.C.; Yuan, B.J. Fuzzy ARIMA model for forecasting the foreign exchange market. Fuzzy Sets Syst. 2001, 118, 9-19. [CrossRef]

26. Abraham, A. Analysis of hybrid soft and hard computing techniques for forex monitoring systems. In Proceedings of the 2002 IEEE World Congress on Computational Intelligence, 2002 IEEE International Conference on Fuzzy Systems, FUZZ-IEEE’02, Proceedings (Cat. No. 02CH37291), Honolulu, HI, USA, 12-17 May 2002; Volume 2, pp. 1616-1621.

27. Neagoe, V.; Iatan, R.; Iatan, H. A nonlinear neuro-fuzzy model for prediction of daily exchange rates. In Proceedings of the World Automation Congress, Seville, Spain, 28 June-1 July 2004; Volume 17, pp. 573-578.

28. Müller, U.A.; Dacorogna, M.M.; Olsen, R.B.; Pictet, O.V.; Schwarz, M.; Morgenegg, C. Statistical study of foreign exchange rates, empirical evidence of a price change scaling law, and intraday analysis. J. Bank. Financ. 1990, 14, 1189-1208. [CrossRef]

29. Zhou, B. High-frequency data and volatility in foreign-exchange rates. J. Bus. Econ. Stat. 1996, 14, 45-52.

30. Batten, J.; Mellor, R.; Wan, V. Foreign exchange risk management practices and products used by Australian firms. J. Int. Bus. Stud. 1993, 24, 557-573. [CrossRef] 
31. Mizuno, T.; Kurihara, S.; Takayasu, M.; Takayasu, H. Analysis of high-resolution foreign exchange data of USD-JPY for 13 years. Physica A 2003, 324, 296-302. [CrossRef]

32. Danielsson, J.; Payne, R. Real trading patterns and prices in spot foreign exchange markets. J. Int. Money Financ. 2002, 21, 203-222. [CrossRef]

33. Nassirtoussi, A.K.; Aghabozorgi, S.; Wah, T.Y.; Ngo, D.C.L. Text mining for market prediction: A systematic review. Expert Syst. Appl. 2014, 41, 7653-7670. [CrossRef]

34. Basha, S.M.; Zhenning, Y.; Rajput, D.S.; Iyengar, N.; Caytiles, D. Weighted fuzzy rule based sentiment prediction analysis on tweets. Int. J. Grid Distrib. Comput. 2017, 10, 41-54. [CrossRef]

35. Eng, M.H.; Li, Y.; Wang, Q.G.; Lee, T.H. Forecast forex with ANN using fundamental data. In Proceedings of the 2008 International Conference on Information Management, Innovation Management and Industrial Engineering, Taipei, Taiwan, 19-21 December 2008; Volume 1, pp. 279-282.

36. Rehman, M.; Khan, G.M.; Mahmud, S.A. Foreign currency exchange rates prediction using cgp and recurrent neural network. IERI Procedia 2014, 10, 239-244. [CrossRef]

37. Kamruzzaman, J.; Sarker, R.A. Forecasting of currency exchange rates using ANN: A case study. In Proceedings of the 2003 International Conference on Neural Networks and Signal Processing, Nanjing, China, 14-17 December 2003; Volume 1, pp. 793-797.

38. Balabanov, T.; Zankinski, I.; Dobrinkova, N. Time series prediction by artificial neural networks and differential evolution in distributed environment. In Proceedings of the International Conference on Large-Scale Scientific Computing, Sozopol, Bulgaria, 6-10 June 2011; pp. 198-205.

39. Dymova, L.; Sevastjanov, P.; Kaczmarek, K. A Forex trading expert system based on a new approach to the rule-base evidential reasoning. Expert Syst. Appl. 2016, 51, 1-13. [CrossRef]

40. Putra, A.R.P.; Permanasari, A.E.; Fauziati, S. I forex trend prediction technique using multiple indicators and multiple pairs correlations DSS: A software design. In Proceedings of the 2016 8th International Conference on Information Technology and Electrical Engineering (ICITEE), Yogyakarta, Indonesia, 5-6 October 2016; pp. 1-5.

41. Achchab, S.; Bencharef, O.; Ouaarab, A. A combination of regression techniques and cuckoo search algorithm for FOREX speculation. In Proceedings of the World Conference on Information Systems and Technologies, Madeira, Portugal, 11-13 April 2017; pp. 226-235.

42. Nassirtoussi, A.K.; Aghabozorgi, S.; Wah, T.Y.; Ngo, D.C.L. Text mining of news-headlines for FOREX market prediction: A Multi-layer Dimension Reduction Algorithm with semantics and sentiment. Expert Syst. Appl. 2015, 42, 306-324. [CrossRef]

43. Seifollahi, S.; Shajari, M. Word sense disambiguation application in sentiment analysis of news headlines: An applied approach to FOREX market prediction. J. Intell. Inf. Syst. 2019, 52, 57-83. [CrossRef]

44. Ranjit, S.; Shrestha, S.; Subedi, S.; Shakya, S. Comparison of algorithms in Foreign Exchange Rate Prediction. In Proceedings of the 2018 IEEE 3rd International Conference on Computing, Communication and Security (ICCCS), Kathmandu, Nepal, 25-27 October 2018; pp. 9-13.

45. Ni, L.; Li, Y.; Wang, X.; Zhang, J.; Yu, J.; Qi, C. Forecasting of forex time series data based on deep learning. Procedia Comput. Sci. 2019, 147, 647-652. [CrossRef]

46. Mańdziuk, J.; Rajkiewicz, P. Neuro-evolutionary system for FOREX trading. In Proceedings of the 2016 IEEE Congress on Evolutionary Computation (CEC), Vancouver, BC, Canada, 24-29 July 2016; pp. 4654-4661.

47. Henrique, B.M.; Sobreiro, V.A.; Kimura, H. Literature review: Machine learning techniques applied to financial market prediction. Expert Syst. Appl. 2019, 124, 226-251. [CrossRef]

48. Raimundo, M.S.; Okamoto, J. SVR-wavelet adaptive model for forecasting financial time series. In Proceedings of the 2018 International Conference on Information and Computer Technologies (ICICT), DeKalb, IL, USA, 23-25 March 2018; pp. 111-114.

49. Taveeapiradeecharoen, P.; Chamnongthai, K.; Aunsri, N. Bayesian compressed vector autoregression for financial time-series analysis and forecasting. IEEE Access 2019, 7, 16777-16786. [CrossRef]

50. Serjam, C.; Sakurai, A. Analyzing predictive performance of linear models on high-frequency currency exchange rates. Vietnam J. Comput. Sci. 2018, 5, 123-132. [CrossRef]

51. Das, S.R.; Mishra, D.; Rout, M. A hybridized ELM-Jaya forecasting model for currency exchange prediction. J. King Saud-Univ.-Comput. Inf. Sci. 2020, 32(2), 345-366. [CrossRef]

52. Chandrinos, S.K.; Lagaros, N.D. Construction of currency portfolios by means of an optimized investment strategy. Oper. Res. Perspect. 2018, 5, 32-44. [CrossRef] 
53. Pradeepkumar, D.; Ravi, V. Forecasting financial time series volatility using particle swarm optimization trained quantile regression neural network. Appl. Soft Comput. 2017, 58, 35-52. [CrossRef]

54. Das, S.R.; Mishra, D.; Rout, M.; others. An optimized feature reduction based currency forecasting model exploring the online sequential extreme learning machine and krill herd strategies. Physica A 2019, 513, 339-370. [CrossRef]

55. Galeshchuk, S.; Mukherjee, S. FOREX Trading Strategy Optimization. In Proceedings of the International Symposium on Distributed Computing and Artificial Intelligence, Porto, Portugal, 21-23 June 2017; pp. 69-76.

56. Das, S.R.; Mishra, D.; Rout, M. A hybridized ELM using self-adaptive multi-population-based Jaya algorithm for currency exchange prediction: An empirical assessment. Neural Comput. Appl. 2019, 31, 7071-7094. [CrossRef]

57. Chou, J.S.; Truong, T.T.H. Sliding-window metaheuristic optimization-based forecast system for foreign exchange analysis. Soft Comput. 2019, 23, 3545-3561. [CrossRef]

58. de Almeida, B.J.; Neves, R.F.; Horta, N. Combining Support Vector Machine with Genetic Algorithms to optimize investments in Forex markets with high leverage. Appl. Soft Comput. 2018, 64, 596-613. [CrossRef]

59. Thu, T.N.T.; Xuan, V.D. Using support vector machine in FoRex predicting. In Proceedings of the 2018 IEEE International Conference on Innovative Research and Development (Icird), Bangkok, Thailand, 11-12 May 2018; pp. 1-5.

60. Özorhan, M.O.; Toroslu, İ.H.; Şehitoğlu, O.T. Short-term trend prediction in financial time series data. Knowl. Inf. Syst. 2019, 61, 397-429. [CrossRef]

61. Özorhan, M.O.; Toroslu, İ.H.; Şehitoğlu, O.T. A strength-biased prediction model for forecasting exchange rates using support vector machines and genetic algorithms. Soft Comput. 2017, 21, 6653-6671. [CrossRef]

62. Dash, R. Performance analysis of an evolutionary recurrent Legendre Polynomial Neural Network in application to FOREX prediction. J. King Saud-Univ.-Comput. Inf. Sci. 2017. [CrossRef]

63. Dash, R. An improved shuffled frog leaping algorithm based evolutionary framework for currency exchange rate prediction. Physica A 2017, 486, 782-796. [CrossRef]

64. Chandrinos, S.K.; Sakkas, G.; Lagaros, N.D. AIRMS: A risk management tool using machine learning. Expert Syst. Appl. 2018, 105, 34-48. [CrossRef]

65. Dash, R. Performance analysis of a higher order neural network with an improved shuffled frog leaping algorithm for currency exchange rate prediction. Appl. Soft Comput. 2018, 67, 215-231. [CrossRef]

66. Hajizadeh, E.; Mahootchi, M.; Esfahanipour, A.; Kh, M.M. A new NN-PSO hybrid model for forecasting Euro/Dollar exchange rate volatility. Neural Comput. Appl. 2019, 31, 2063-2071. [CrossRef]

67. Fan, M.H.; Chen, M.Y.; Liao, E.C. A deep learning approach for financial market prediction: Utilization of Google trends and keywords. Granul. Comput. 2019, 1-10. [CrossRef]

68. Koskivaara, E. Artificial Neural Networks in Auditing: State of the Art; Technical Report; Turku Centre for Computer Science: Truku, Finland, 2003.

69. Ravi, V.; Pradeepkumar, D.; Deb, K. Financial time series prediction using hybrids of chaos theory, multi-layer perceptron and multi-objective evolutionary algorithms. Swarm Evol. Comput. 2017, 36, 136-149. [CrossRef]

70. Lee, R.S. COSMOS trader-Chaotic Neuro-oscillatory multiagent financial prediction and trading system. J. Financ. Data Sci. 2019, 5, 61-82. [CrossRef]

71. Pradeepkumar, D.; Ravi, V. FOREX Rate Prediction: A Hybrid Approach Using Chaos Theory and Multivariate Adaptive Regression Splines. In Proceedings of the 5th International Conference on Frontiers in Intelligent Computing: Theory and Applications; Satapathy S., Bhateja V., Udgata S., Pattnaik P., Eds.; Springer: Singapore, 2017; Volume 515, pp. 219-227.

72. Lee, R.S. Chaotic Interval Type-2 Fuzzy Neuro-oscillatory Network (CIT2-FNON) for Worldwide 129 Financial Products Prediction. Int. J. Fuzzy Syst. 2019, 21, 2223-2244. [CrossRef]

73. Contreras, A.V.; Llanes, A.; Pérez-Bernabeu, A.; Navarro, S.; Pérez-Sánchez, H.; López-Espín, J.J.; Cecilia, J.M. ENMX: An elastic network model to predict the FOREX market evolution. Simul. Model. Pract. Theory 2018, 86, 1-10. [CrossRef]

74. Carapuço, J.; Neves, R.; Horta, N. Reinforcement learning applied to Forex trading. Appl. Soft Comput. 2018, 73, 783-794. [CrossRef]

75. Bartoš, E.; Pinčák, R. Identification of market trends with string and D2-brane maps. Physica A 2017, 479, 57-70. [CrossRef] 
76. Taveeapiradeecharoen, P.; Aunsri, N. Dynamic model averaging for daily forex prediction: A comparative study. In Proceedings of the 2018 International Conference on Digital Arts, Media and Technology (ICDAMT), Phayao, Thailand, 25-28 February 2018; pp. 321-325.

77. Wilinski, A. Time series modeling and forecasting based on a Markov chain with changing transition matrices. Expert Syst. Appl. 2019, 133, 163-172. [CrossRef]

78. Fiszeder, P.; Fałdziński, M. Improving forecasts with the co-range dynamic conditional correlation model. J. Econ. Dyn. Control. 2019, 108, 103736. [CrossRef]

79. Goumatianos, N.; Christou, I.T.; Lindgren, P.; Prasad, R. An algorithmic framework for frequent intraday pattern recognition and exploitation in forex market. Knowl. Inf. Syst. 2017, 53, 767-804. [CrossRef]

80. Petropoulos, A.; Chatzis, S.P.; Siakoulis, V.; Vlachogiannakis, N. A stacked generalization system for automated FOREX portfolio trading. Expert Syst. Appl. 2017, 90, 290-302. [CrossRef]

81. Zhu, J.; Wu, X.; Xiao, J.; Huang, C.; Tang, Y.; Deng, K. Improved expert selection model for forex trading. Front. Comput. Sci. 2018, 12, 518-527. [CrossRef]

82. Pushpa, P. Context Information based FOREX services. In Proceedings of the EAI International Conference on Ubiquitous Communications and Network Computing, Bangalore, India, 3-5 August 2017; pp. 79-90.

83. Weerathunga, H.; Silva, A. DRNN-ARIMA Approach to Short-term Trend Forecasting in Forex Market. In Proceedings of the 2018 18th International Conference on Advances in ICT for Emerging Regions (ICTer), Colombo, Sri Lanka, 26-29 September 2018; pp. 287-293.

(C) 2020 by the authors. Licensee MDPI, Basel, Switzerland. This article is an open access article distributed under the terms and conditions of the Creative Commons Attribution (CC BY) license (http:/ / creativecommons.org/licenses/by/4.0/). 